\title{
Association of self-reported symptoms with serum levels of vitamin $D$ and multivariate cytokine profile in healthy women
}

This article was published in the following Dove Press journal:

Journal of Inflammation Research

17 March 2017

Number of times this article has been viewed

\author{
Fawaz Y Azizieh' \\ Khulood O Alyahya ${ }^{2}$ \\ Kamaludin Dingle' \\ 'Department of Mathematics and \\ Natural Sciences, Gulf University for \\ Science and Technology, Internationa \\ Centre for Applied Mathematics and \\ Computational Bioengineering, West \\ Mishref, Kuwait; ${ }^{2}$ Science Department, \\ College of Basic Education, Public \\ Authority for Applied Education and \\ Training, Kuwait City, Kuwait
}

Background: Although a large number of studies have investigated possible relationships among serum levels of vitamin D or cytokines with disease progress and prognosis, similar studies on self-reported symptoms are still controversial. The overall objective of this study was to look into the association between serum levels of vitamin D or cytokines with self-reported symptoms related to musculoskeletal pain, sleep disorders, and premenstrual syndrome (PMS) in healthy adult women.

Subjects and methods: Venous blood samples were collected from 117 healthy adult women, and serum levels of vitamin D, pro-inflammatory cytokines (IL-1 $\beta$, IL-6, IL-8, IL-17, IFN- $\gamma$, and TNF- $\alpha$ ) and anti-inflammatory cytokines (IL-4, IL-10, and IL-13) were measured. Groups were tested for differences in single parameters, pro-:anti-inflammatory cytokine ratios, and differences in multivariate patterns.

Results: There were no significant associations between serum levels of vitamin D and any of the self-reported symptoms studied. However, serum levels of certain pro-inflammatory cytokines were significantly higher in subjects with musculoskeletal pain (IL-8, $P=0.008$ ), sleep disorders (IFN- $\gamma, P=0.02$ ), and PMS (IL- 8 and TNF- $\alpha, P=0.009$ and 0.002 , respectively) compared to subjects who reported no symptoms. The pro-:anti-inflammatory cytokine ratios showed proinflammatory cytokine dominance in subjects with self-reported symptoms, particularly in the groups with deficient levels of vitamin D. However, the multivariate cytokine-pattern analysis was significantly different between PMS groups only.

Conclusion: These data point to a possible role of pro-inflammatory cytokines as a contributing factor in self-reported symptoms related to musculoskeletal pain, sleep disorders, and PMS.

Keywords: vitamin D, cytokines, adult women, self-reported symptoms, inflammation

\section{Introduction}

While healthy levels of vitamin D were initially thought to be essential mainly for bone health, a variety of nonskeletal diseases have been found to be associated with deficient levels. The range includes almost all organ systems, such as disorders of the gastrointestinal, cardiovascular, muscular, and immune systems. ${ }^{1-3}$ The list is neverending, and has recently been extended to include psychological health, behavior disorders, and brain functioning. Deficient levels of vitamin D have been associated with anxiety, low mood, impaired cognitive performance, and depression. ${ }^{4,5}$ Moreover, overwhelming scientific evidence suggests that maintenance of an adequate vitamin D level is essential in the prevention of a wide variety of health disorders, ${ }^{6-9}$ and is thought to be one of the key parameters contributing to healthy immune-system function and inflammatory responses. ${ }^{10-12}$
Correspondence: Fawaz Y Azizieh Department of Mathematics and Natural Sciences, Gulf University for Science and Technology, Building I, Block 5, Mubarak Al-Abdullah, West Mishref, Kuwait

Tel + 96525307499

Email Azizieh.F@gust.edu.kw 
Cytokines are messengers of the immune system that are involved in many physiological and pathological processes. Pro-inflammatory cytokines such as interleukin (IL)-6, IL-8, IL-17, tumour necrosis factor-alpha (TNF- $\alpha$ ) and interferon-gamma (IFN- $\gamma$ ) promote inflammation, while anti-inflammatory cytokines, such as IL-4, IL-10, and IL-13, reduce inflammation and promote healing. ${ }^{13,14}$ An imbalance or excessive production of these cytokines has been linked to different diseases and symptoms, ranging from fever to death and atherosclerosis to cancer. ${ }^{10-16}$ Dysregulation of pro-inflammatory cytokines has also been linked to sickness behavior, such as pain perception, cognitive impairment, depression, and other neurologically related effects. ${ }^{13,14,17}$ It has been further suggested to use serum levels of cytokines as potential biomarkers in diseases, such as in rheumatoid arthritis. ${ }^{18}$ However, as pathology is because of an imbalance in the network, a single biomarker may be insufficient, and it has been further proposed to use ratios of two cytokines or develop a multivariate "cytokine signature" or score, where different combinations are used for better achieving a prediction of marking and providing better understanding. ${ }^{19}$ This paper is a contribution to the understanding of possible associations between serum levels of vitamin $\mathrm{D}$ and cytokines with self-reported symptoms/health complaints related to musculoskeletal pain, sleep disorders, and premenstrual syndrome (PMS) in healthy adult women.

Musculoskeletal pain is one of the most common types of pain, and can range from a simple ache to long-lasting pain in one or multiple locations. Several studies have reported the prevalence of severe hypovitaminosis D in patients with persistent, nonspecific self-reported musculoskeletal pain, ${ }^{2,20}$ which was relieved in the majority of patients by vitamin $\mathrm{D}$ supplementation. ${ }^{21-23}$ Certain pro-inflammatory cytokines, such as IL-1 $\beta$, IL- 6 , and TNF- $\alpha$ have also been suggested to be involved in the process of pathological pain. ${ }^{14,17}$

Sleep disorders include poor sleep quality, fragmented sleep, restricted sleep, and variability in bed and wake times. It has been hypothesized that the world epidemic of sleep disorders is linked to vitamin D deficiency, ${ }^{24}$ while vitamin D supplementation has been reported to improve sleep quality. ${ }^{22}$ It has further been reported that high levels of circulating inflammatory cytokines (e.g. IL-1 $\beta$, IL-6, IL-8, TNF- $\alpha$, and IFN- $\gamma$ ) are associated with sleep disorders. ${ }^{25-27}$

It is estimated that up to $85 \%$ of menstruating women report having one or more symptoms of PMS. ${ }^{28}$ However, their severity may range from just slightly noticeable all the way to intense, and range to include a wide variety of emotional, behavioral, and physical signs and symptoms. ${ }^{28}$ Although the etiology remains unknown, few studies have associated PMS with low levels of vitamin $\mathrm{D},{ }^{29,30}$ and others have reported positive associations between serum levels of inflammatory markers and PMS symptoms in young women. ${ }^{31,32}$

Though some of the research just cited supports a potential association between vitamin D deficiency and cytokines in reported symptoms of musculoskeletal pain, sleep disorders, and PMS, there is another body of evidence that questions such associations. ${ }^{33-37}$ In summary, studies in this field are still controversial, and further research is needed to address possible associations in different populations and age-groups. Given this background, our overall objective was to study possible associations between self-reported symptoms presented by healthy adult Kuwaiti women and serum levels of vitamin $D$ and selected combinations of proinflammatory (IL-1 $\beta$, IL-6, IL-8, IL-17, IFN- $\gamma$, and TNF- $\alpha$ ) and anti-inflammatory (IL-4, IL-10, and IL-13) cytokines.

\section{Subjects and methods Subjects}

Invitation letters were sent to the mothers of students from a previous study ${ }^{38}$ who were recruited from schools located in each governorate in Kuwait. In addition, female students in the College of Basic Education, Public Authority for Applied Education and Training, Kuwait were also invited to participate. A total of 117 healthy adult women aged 19-47 years were recruited from December 2011 to March 2012. In order to limit the effect of the heterogeneous composition of the study population in terms of variation in ethnicity, race, lifestyle, and diet, this study included Kuwaiti citizens only. All subjects were nonpregnant, nonbreastfeeding, free from infections/chronic diseases, and not taking any medications or vitamin/mineral supplements/injections for the previous 6 months. All participants provided informed consent, and were included in the study as apparently healthy individuals. The study was granted the ethical approval of the Joint Committee for the Protection of Human Subjects in Research of the Kuwait Institute for Medical Specialization, Ministry of Health and the Abdul Mohsen al Abdul Razzaq Health Sciences Center, Kuwait University.

All participants were interviewed and physically examined for their weight, height, and body mass index (BMI). A self-reported questionnaire (SRQ) was administered by an interviewer to gather information about their general health, but more specifically the presence of any health complaints or symptoms over the past 6 months. A list of common symptoms, mostly physical, were covered, and among these only sleeping disorders, PMS, and musculoskeletal pain were studied in this context. 
For sleeping disorders, participants were asked the question "Do you have trouble falling asleep or staying asleep?", and for PMS symptoms the question was "Do you suffer from premenstrual symptoms?". Participants were requested to respond to these questions by choosing from "no symptoms", "sometimes", or "most of the time", which were subsequently coded as 0,5 , and 10 , respectively. With regard to musculoskeletal pain, the question asked was "Do you suffer from any pain? If yes, where?” Also, participants were requested to rate the severity of pain from 0 (no complaint) to 10 (highest frequency and intensity). Shoulder pain was the pain most associated with the biomarkers in this study, and hence was included. It was referred to as musculoskeletal pain. Due to a weak association between the severity of symptoms and the biomarkers, all of the responses to the three symptoms were recategorized into binary scales: yes (symptomatic) and no (asymptomatic).

\section{Blood sampling}

Venous blood samples were collected into Vacutainer tubes and allowed to clot at room temperature for 30 minutes. The coagulated blood was centrifuged for 10 minutes at 3,000 $\times g$; the serum was aliquoted into sterile tubes and stored frozen till the date of analysis.

\section{Measurement of vitamin D}

Quantitation of serum 25(OH)D was performed with a commercial radioimmunoassay kit (Immunodiagnostic Systems Holdings PLC, Boldon Colliery, UK) that detects 25(OH)D in the range of 5-300 nmol/L with inter- and intra-assay coefficients of variation of $3.43 \%$ and $5.44 \%$, respectively. The cutoff to define an indeficient circulating concentration of $25(\mathrm{OH}) \mathrm{D}$ is taken to be $25 \mathrm{nmol} / \mathrm{L}$, and concentrations lower than that are categorized as deficient. ${ }^{3,39}$ Subjects were thus classified as not deficient (ND) if their serum vitamin D level was $\geq 25 \mathrm{nmol} / \mathrm{L}$ or deficient (D) if their level was $<25 \mathrm{nmol} / \mathrm{L}$.

\section{Cytokine analysis}

A multiplex enzyme-linked immunosorbent assay containing dyed microspheres conjugated with a monoclonal antibody specific for a target protein was used in accordance with the manufacturer's instructions (Merck Millipore, Billerica, MA, USA). Soluble molecules were measured using commercially available human cytokine kits (HCytoMag-60K) 9-plex panel: IL-1 $\beta$, IL-4, IL-6, IL-8, IL-10, IL-17, IL-13, IFN- $\gamma$, and TNF- $\alpha$. Each experiment was performed in duplicate, using the same procedure described by the manufacturer.
Serum concentrations of all cytokines were determined using a MagPix array reader (Luminex, Austin, TX, USA), which quantifies multiplex immunoassays in a 96-well format with very small sample volumes. The analyte concentration was calculated using a standard curve, with software provided by the manufacturer (Luminex Manager). The detection sensitivity of the assays was $0.7 \mathrm{pg} / \mathrm{mL}$ of TNF- $\alpha, 0.8 \mathrm{pg} /$ $\mathrm{mL}$ of IL-1 $\beta$, IL-4, IL-6, IL-8, IL-13, IL-17, and IFN- $\gamma$, and $1.1 \mathrm{pg} / \mathrm{mL}$ of IL-6. Quality-control measures were followed as per the recommendation of the manufacturers. The range of intra-assay coefficients of variation was $1.6 \%-2.9 \%$ while that of interassay coefficients of variation was $9.2 \%-18.3 \%$. Accuracy in terms of recovery in matrix was $94.5 \%-104 \%$.

\section{Statistical methods}

Statistical analysis was done using SPSS 23 software (IBM, Armonk, NY, USA) and Python using IPython. ${ }^{40}$ Normality distribution of data was first determined by KolmogorovSmirnov test. As data were found not to be normally distributed, nonparametric analysis was used throughout the analysis (including the box-and-whisker plots). For comparing groups with small sample numbers $(<20)$, Monte Carlo sampling was used to obtain $P$-values for Mann-Whitney $U$ test statistics, rather than the default $P$-values (which assume large sample sizes in both groups). Spearman's rank correlation coefficient was calculated to determine correlations between complaint scores and cytokine levels. For testing multivariate statistical differences between the groups, the nonparametric multivariate Cramer test ${ }^{41}$ was run in RStudio. ${ }^{42}$ Prior to running this test, the multivariate data were standardized by reducing skew, scaling by the mean, and subtracting the mean of each variable. Linear and univariate multiple regression models were used to determine the relationships among serum $25(\mathrm{OH}) \mathrm{D}$, various cytokines, and self-reported symptoms. $P$-values less than 0.05 were considered statistically significant for all tests.

\section{Results}

Table 1 presents the basic demographic data from all healthy women enrolled in the study. Subjects were grouped into symptomatic (denoted by $\mathrm{S}$; those who self-reported a score $\geq 5$ ) or not symptomatic (denoted by NS; those who self-reported a score of $<5$ ). Among the 117 subjects, only $21 \%(24 / 117)$ reported a median musculoskeletal pain score of 6 , while the others reported no similar pain. Those who expressed this pain were statistically significantly younger in age compared to the group of no pain $(P=0.027)$. Both groups were similar in height, weight, and BMI (Table 1). 
Table I Demographic characteristics of women enrolled in the study (median)

\begin{tabular}{|c|c|c|c|c|c|c|}
\hline $\begin{array}{l}\text { Self-reported } \\
\text { symptoms }\end{array}$ & n (\%) & Symptom score & Age (years) & Height (cm) & Weight (kg) & BMI $\left(\mathrm{kg} / \mathrm{m}^{2}\right)$ \\
\hline \multicolumn{7}{|c|}{ Musculoskeletal pain } \\
\hline$S$ & $24(2 I)$ & 6 & 24.5 & 161 & 67 & 25.9 \\
\hline$N$ & $93(79)$ & 0 & 30 & 159 & 68 & 27.1 \\
\hline & & $<0.0001$ & 0.027 & 0.07 & 0.49 & 0.27 \\
\hline \multicolumn{7}{|l|}{ Sleep disorders } \\
\hline$S$ & $59(50)$ & 10 & 28 & 159 & 67 & 26.6 \\
\hline $\mathrm{N}$ & $58(50)$ & 0 & 27 & 159 & 68 & 26.9 \\
\hline & & $<0.0001$ & 0.48 & 0.49 & 0.41 & 0.39 \\
\hline \multicolumn{7}{|l|}{ PMS } \\
\hline $\mathrm{S}$ & $94(80)$ & 10 & 28.5 & 159 & 67 & 26.6 \\
\hline NS & $23(20)$ & 0 & 25 & 159 & 67 & 26.7 \\
\hline & & $<0.0001$ & 0.16 & 0.41 & 0.46 & 0.46 \\
\hline
\end{tabular}

Abbreviations: BMI, body mass index; S, symptomatic (subjects who self-reported a score $\geq 5$ ); NS, not symptomatic (subjects who self-reported a score $<5$ ); PMS, premenstrual syndrome.

Almost half of the subjects, however, reported sleep disorders (59 of 117), and both groups were comparable in other demographic data. With respect to PMS, 80\% (94 of 117) of women in the study group reported a median score of 10 compared to only $20 \%$ (23 of 117) with no such complaint. Similarly, both groups were comparable in age, height, weight, and BMI (Table 1). No significant association between BMI and levels of vitamin D was noted in our samples.

\section{Levels of vitamin D and pro- and anti-inflammatory cytokines}

Table 2 compares serum levels of vitamin D and pro- and anti-inflammatory cytokines with respect to self-reported symptoms of musculoskeletal pain, sleep disorders, and PMS. Subjects were grouped as S or NS. It was worth mentioning that IL-6, IL-10, and IL-13 were not measurable in any of the sera tested.

There were no significant differences in serum levels of vitamin D among subjects with or without self-reported symptoms of musculoskeletal pain, sleep disorders, or PMS.
However, serum levels of certain pro-inflammatory cytokines were significantly different between the groups. Compared to the groups who reported no symptoms, subjects with musculoskeletal pain had higher levels of IL-8 $(P=0.03)$, subjects with sleep disorders had higher levels of IFN- $\gamma(P=0.02)$, and subjects with PMS had higher levels of IL- 8 and TNF- $\alpha$ $(P=0.009$ and 0.0002 , respectively) (Table 2$)$. These data point to a possible role of some pro-inflammatory cytokines as a contributing factor in these self-reported symptoms. It was worth mentioning that among the pro-inflammatory cytokines measured, serum levels of IFN- $\gamma$ correlated significantly with levels of IL-8 and IL-17 (Spearman's $r_{\mathrm{s}}=0.49$ and 0.61, respectively; $P<0.0001)$; similarly, serum levels of IL-8 correlated with IL-17 (Spearman's $r_{\mathrm{s}}=0.49, P<0.0001$ ).

We further grouped subjects based on the serum level of vitamin $\mathrm{D}$ into not deficient (subjects with vitamin $\mathrm{D}$ level $\geq 25 \mathrm{nmol} / \mathrm{L}$, denoted by ND, $\mathrm{n}=28$ [24\%]) and deficient (subjects with vitamin $\mathrm{D}$ level $<25 \mathrm{nmol} / \mathrm{L}$, denoted by $\mathrm{D}, \mathrm{n}=89[76 \%])$. Subjects with deficient serum levels of vitamin $\mathrm{D}$ were statistically significantly younger than those with indeficient levels $(P=0.004)$. However, there were no

Table 2 Median serum levels of vitamin D and pro- and anti-inflammatory cytokines in symptomatic and asymptomatic subjects

\begin{tabular}{|c|c|c|c|c|c|c|c|c|c|}
\hline \multirow[t]{3}{*}{$\mathbf{n}$} & \multicolumn{2}{|c|}{ Musculoskeletal pain } & \multirow[t]{3}{*}{$P$-value } & \multicolumn{2}{|c|}{ Sleep disorders } & \multirow[t]{3}{*}{$P$-value } & \multicolumn{2}{|l|}{ PMS } & \multirow[t]{3}{*}{$P$-value } \\
\hline & $\mathbf{S}$ & NS & & $\mathbf{S}$ & NS & & $\mathbf{S}$ & NS & \\
\hline & 24 & 93 & & 59 & 58 & & 94 & 23 & \\
\hline Vitamin D (nmol/L) & 15 & 17 & 0.18 & 16 & 17 & 0.47 & 17 & 14 & 0.30 \\
\hline \multicolumn{10}{|l|}{ Cytokine (pg/mL) } \\
\hline IL-I $\beta$ & 0.4 & 0.2 & 0.82 & 0.4 & 0.03 & 0.26 & 0.3 & 0.2 & 0.32 \\
\hline IL-8 & 16.9 & 13.7 & 0.03 & 14.9 & 13.3 & 0.27 & 14.5 & 10.7 & 0.009 \\
\hline IL-17 & 5.1 & 3.6 & 0.08 & 5.4 & 3.5 & 0.08 & 4 & 4.3 & 0.47 \\
\hline $\mathrm{IFN}-\gamma$ & 12.9 & 10.2 & 0.39 & 14.3 & 9.7 & 0.02 & 11 & 10.7 & 0.97 \\
\hline TNF- $\alpha$ & 10.6 & 9.8 & 0.32 & 10.6 & 9.8 & 0.47 & 10.6 & 7.6 & 0.002 \\
\hline IL-4 & 171.7 & 142.4 & 0.98 & 153.3 & 150 & 0.95 & 163.9 & 35.2 & 0.18 \\
\hline
\end{tabular}

Abbreviations: PMS, premenstrual syndrome; , symptomatic (subjects who self-reported a score $\geq 5$ ); NS, not symptomatic (subjects who self-reported a score $<5$ ). 
statistical significant differences between the two groups with respect to self-reported complaints of musculoskeletal pain, sleep disorders, or PMS (data not shown).

For each of the complaints under study (musculoskeletal pain, sleep disorders, and PMS), we further stratified the subjects and compared cytokine levels in the following four groups: group 1, subjects who self-reported symptoms and had indeficient levels of vitamin $\mathrm{D}$ (denoted by $\mathrm{S} / \mathrm{ND}, \mathrm{n}=4,13$, and 21 for musculoskeletal pain, sleep disorders, and PMS, respectively); group 2, subjects who reported no symptoms and had indeficient levels of vitamin D (denoted by NS/ND, $\mathrm{n}=24,15$, and 7 , respectively), group 3 , subjects who selfreported symptoms and had deficient levels of vitamin D (denoted by S/D, n=20, 46, and 73, respectively), and group 4, subjects who reported no symptoms and had deficient levels of vitamin $\mathrm{D}$ (denoted by NS/D, n=69, 43, and 16, respectively).

Figure 1 presents median serum levels of IL-8, IL-17, IFN- $\gamma$, and TNF- $\alpha$ in the stratified subject groups. With regard to musculoskeletal pain, significantly higher levels of IL-8 and IL-17 were present in sera of subjects in the S/D group compared to subjects in the NS/D group $(P=0.009$ and 0.031, respectively) (Figure 1A and B). With respect to sleep disorders, women in the S/ND group had significantly higher serum levels of IL-17 and IFN- $\gamma$ compared to subjects in the NS/ND group ( $P=0.017$ and 0.003 , respectively) (Figure 1B and $\mathrm{C})$. For PMS, women in the S/ND group had significantly higher serum levels of IL-8 compared to subjects in the NS/ND group $(P=0.006)$ (Figure 1A), and subjects in the $\mathrm{S} / \mathrm{D}$ group had significantly higher levels of IL-17 and TNF- $\alpha$ compared to subjects in the NS/D group $(P=0.039$ and 0.004 , respectively) (Figure 1B and D). There were no statistical significant differences among the four groups with respect to serum levels of IL-1 $\beta$ and IL-4 (data not shown).

\section{Pro-:anti-inflammatory cytokine ratio and multivariate profiles in symptomatic and asymptomatic healthy subjects}

Absolute levels of cytokines per se may not be as informative as relative levels of pro-:anti-inflammatory cytokines, as this will provide a better picture of the overall dominance of one group of cytokines over another. As anti-inflammatory cytokines may neutralize or antagonize the effect of pro-inflammatory cytokines, it would be logical to calculate ratios of pro-inflammatory to anti-inflammatory cytokines as an indicator of the overall cytokine bias, and thus the overall immunoreactivity. With this in mind, we calculated the ratios of pro-:anti-inflammatory cytokines in different permutations.

Table 3 presents ratios of the pro-inflammatory cytokines (TNF- $\alpha$, IFN- $\gamma$, IL-8, and IL-17) to the anti-inflammatory cytokine (IL-4) in all three self-reported symptoms under study. Among subjects with indeficient levels of vitamin D, women with sleep disorders had higher ratios of all pro-:antiinflammatory cytokines compared to those with no symptoms (NS/D). However, among women with musculoskeletal pain and PMS, only two of the four permutations of ratios showed a similar trend.

On the other hand, among subjects with deficient vitamin D levels, women with symptomatic musculoskeletal pain, sleep disorders, and PMS (S/D) had consistently higher ratios of all pro-:anti-inflammatory cytokine combination compared to those with no symptoms (NS/D). The only exception was the IL-17:IL-4 ratio, which was equal in women with or without sleep-disorder complaints. These data further point to a possible pro-inflammatory cytokine dominance associated with self-reported symptoms of musculoskeletal pain, sleep disorders, and PMS, particularly in subjects with deficient levels of vitamin D.

Given the fact that cytokines are part of a large network, it is rational to study the combined pattern of all measured cytokines. We thus analyzed our data with the multivariate statistical Cramer method, which can detect more complex pattern differences than simple differences in individual levels or ratios. It is useful to employ such multivariate methods, because it is in principle possible that none of the individual variables shows statistically significant differences in median levels when comparing samples of two groups, while a multivariate method may nonetheless uncover a difference in the multivariate pattern. On the other hand, the ability to detect multivariate differences comes at the expense of a reduced ability to detect individual cytokine differences.

The result of the Cramer test showed a statistically significant multivariate cytokine-pattern difference between the $\mathrm{S}$ and NS groups for PMS $(P=0.026)$. However, no similar significant differences were found for the other two symptoms (musculoskeletal pain and sleep disorders). Applying the same statistical test after stratifying the groups based on serum levels of vitamin D, we found no statistical significant differences in the cytokines patterns in S/ND vs NS/ND or $\mathrm{S} / \mathrm{D}$ vs NS/D, despite finding several individual cytokine differences, as depicted in Figure 1.

\section{Discussion}

Several studies have reported positive associations between serum levels of vitamin D or its supplementation and well-being, ${ }^{1-9,21-23}$ while others have shown a minor or no relation. ${ }^{33-35}$ The controversy goes further to question if vitamin D deficiency has anything to do with patients' complaints 

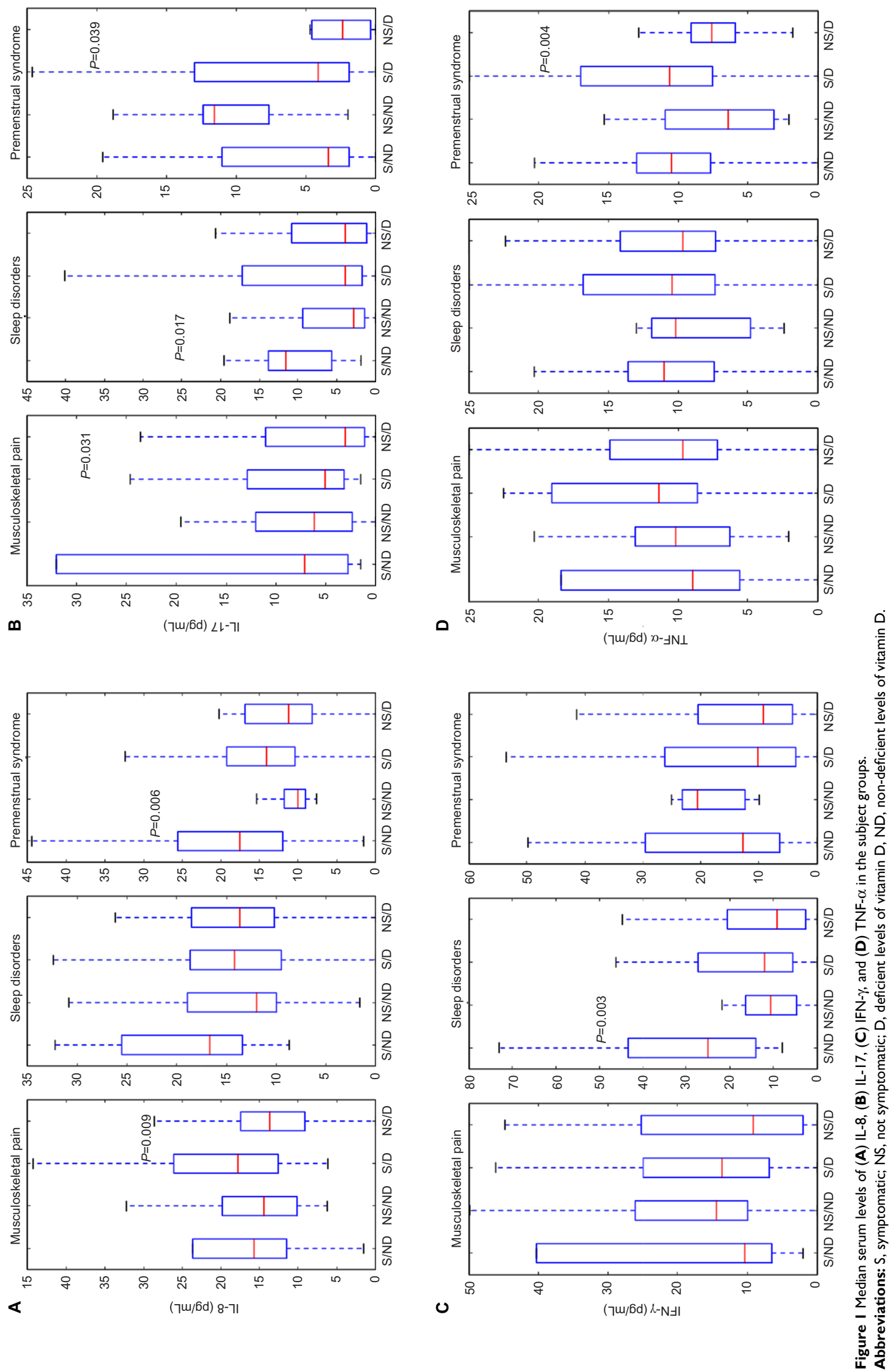
Table 3 Ratio of pro-:anti-inflammatory cytokines in all groups

\begin{tabular}{lllll}
\hline Group & TNF- $\alpha$ /IL-4 & IFN- $\gamma$ /IL-4 & IL-8/IL-4 & IL-I 7/IL-4 \\
\hline \multicolumn{2}{l}{ Musculoskeletal pain } & & & \\
S/ND & 9 & 10.4 & 15.8 & 7.1 \\
NS/ND & 10.2 & 14.4 & 14.5 & 6.2 \\
S/D & 11.4 & 13.7 & 17.8 & 5.1 \\
NS/D & 9.7 & 9.1 & 13.7 & 3 \\
Sleep disorders & & & \\
S/ND & 11 & 25 & 16.7 & 11.6 \\
NS/ND & 10.2 & 10.7 & 12 & 2.8 \\
S/D & 10.4 & 12.1 & 14.2 & 4 \\
NS/D & 9.7 & 9.1 & 13.7 & 4 \\
PMS & & & & \\
S/ND & 10.5 & 12.7 & 17.6 & 3.4 \\
NS/ND & 6.5 & 20.6 & 10.1 & 11.6 \\
S/D & 10.6 & 10.2 & 14.1 & 4.1 \\
NS/D & 7.6 & 9.3 & 11.3 & 2.4 \\
\hline
\end{tabular}

Abbreviations: $S$, symptomatic; NS, not symptomatic; $D$, deficient levels of vitamin D; ND, non-deficient levels of vitamin D.

and symptoms, or whether these are simply related to poor diet and reduced sunlight exposure. ${ }^{36}$

On the contrary, it is well documented that excess cytokines can bring on flu-like symptoms, including fever, pain, and fatigue, while administering certain ones results in temporary symptoms that patients did not normally have before. ${ }^{14,17}$ Some cytokines, such as IL-1 $\beta$, IL-6, and TNF- $\alpha$, are known to increase substance $P$, which transmits pain messages. ${ }^{14,17}$ Among the common symptoms in any community are those related to musculoskeletal pain, sleep disorders, and PMS. While these symptoms are nonspecific and could be attributed to any of a number of other medical comorbidities, our interest was to study these complaints and investigate any possible association with serum levels of vitamin $D$ and pro- and anti-inflammatory cytokines in the serum samples of healthy adult women.

There have been a number of SRQs to address generic health status with proven reliability and validity. The 20-question SRQ-20 developed by the World Health Organization is an example. This questionnaire has been widely used in both research and medical practice to evaluate mental disorders in healthy individuals. ${ }^{43,44}$ Another example is the Short Form questionnaire (SF-36), developed by the Boston Health Research Institute, which comprises 36 questions to assess quality of life and is an instrument that relies greatly on the individual's perception and personal judgment. This has also been validated, and is widely used in research and by health care organizations. ${ }^{45,46}$ Further, a single self-rated health question has also been validated in population-based studies. ${ }^{47,48}$ With four response categories, one question revealed significant associations with lifestyle factors and objective health status.
Despite the controversy in reliability of self-reported data, a lot of gathered information in research is in fact self-reported. Examples include the individual's residence, education, marital status, lifestyle factors, physical activity, food consumption, and even emotional status. ${ }^{44,48}$ Although research has shown that people tend to overestimate or underestimate reporting variables, such as the amount of food they usually consume, ${ }^{49}$ other self-reported information, such as smoking or food consumption, is acceptable and trusted. ${ }^{48,50}$ Because a wide range of health problems have been linked to both low levels of serum vitamin $\mathrm{D}$ and certain inflammatory markers, including muscle pain, ${ }^{2,20} \mathrm{PMS},{ }^{29-31}$ and sleeping disorders, ${ }^{24}$ it was worth assessing the association among these variables, if any, based on an easily administered and convenient tool, such as a broad SRQ.

There has been no absolute agreement on the optimal level of serum vitamin $\mathrm{D}$ to date. The World Health Organization and the Nordic Council of Ministers ${ }^{39}$ agreed on marking sufficiency at $50 \mathrm{nmol} / \mathrm{L}$, but defined deficiency as below $25 \mathrm{nmol} / \mathrm{L}$. Ranges of $25-50 \mathrm{nmol} / \mathrm{L}$ remain controversial, and are reported as insufficient levels. ${ }^{51}$ In this study, $25 \mathrm{nmol} / \mathrm{L}$ was a more reasonable cutoff for the data set we had. In addition, only a few subjects obtained serum levels of vitamin $\mathrm{D}$ of $25-50 \mathrm{nmol} / \mathrm{L}$.

The present study did not find significant correlations among serum level of vitamin D and any of the studied selfreported symptoms. We predicted that subjects with deficient serum levels of vitamin D would have higher complaint scores compared to subjects with indeficient levels; ;,20-24,29 however, we did not find vitamin D levels to be significantly different between the two groups studied. This is in line with other studies that have not supported such association. ${ }^{33-36}$ The controversy is so ample, as many factors may contribute to the wide discrepancy. These may include, but are not limited to, the criteria of inclusion, duration of symptoms, lack of control group, seasonal variation, ethnic homogeneity of populations studied, and many others. No doubt, more studies with larger samples are required to confirm these findings.

Much has been written about the association of proinflammatory cytokines and sickness behavior, ${ }^{14,17-19}$ with similar broad controversy. ${ }^{37}$ Our data showed that subjects with musculoskeletal pain, sleep disorders, and PMS had statistically significantly higher serum levels of pro-inflammatory cytokines (IL-8, IFN- $\gamma$, IL-8, and TNF- $\alpha$ ) compared to subjects who did not report similar pain. As there is increasing evidence that vitamin $\mathrm{D}$ has regulatory effects on cytokine production, ${ }^{10-12}$ this encouraged us to stratify our initial grouping of $\mathrm{S}$ versus NS further into subgroups based on serum levels of vitamin D: D versus ND. This resulted in four subgroups: S/ND, NS/ND, S/D, and NS/D. 
Among ND subjects, and with regard to symptoms related to sleeping disorders and PMS, S/ND subjects had significantly higher pro-inflammatory cytokine (IFN- $\gamma$, IL-17) levels and IL-8 compared to asymptomatic ones (NS/ ND). Similarly, groups with deficient vitamin D levels, and with regard to symptoms related to musculoskeletal pain and PMS, S/D subjects had significantly higher pro-inflammatory cytokine levels of IL-8, IL-17, and TNF- $\alpha$ compared to NS/D subjects (Figure 1). This supports a potential association of pro-inflammatory cytokines with the respective symptoms.

While IFN- $\gamma$ and TNF- $\alpha$ play well-established key roles in pro-inflammatory responses, ${ }^{13,18,19} \mathrm{IL}-8$ is known to induce chemotaxis. ${ }^{17}$ We further reported significant correlation among serum levels of IFN- $\gamma$, IL-8, and IL-17, which is consistent with what was reported by others. ${ }^{15,16}$ It has not yet been determined, however, whether elevated cytokines are the direct cause of the symptoms or merely secondary to another factor. Circulating cytokine levels present a tip of an iceberg to those produced in tissues at local concentrations. Further, these levels may increase up to 1,000 times in different situations, including nonspecific subclinical infections or inflammations. Levels may also vary due to patient factors, such as age, sex, circadian rhythms, food intake, exercise, stress, other immune problems, abnormal hormone activity, or sleep disturbances..$^{18}$ For example, elevated cytokine levels can be induced through sleep deprivation, and it has been suggested that disordered sleep could be the real root of many symptoms. ${ }^{22,26,27}$

There are many publications in the literature that propose cytokine levels as potential biomarkers of several chronic and acute inflammatory diseases. With rheumatoid arthritis as an example, pro-inflammatory cytokines, such as IL-6, TNF- $\alpha$, and IL-17, are currently considered potential biomarkers. ${ }^{52,53}$ Further, levels of IL- 6 and IL-17 have been reported to be associated with disease activity, ${ }^{53} \mathrm{GM}-\mathrm{CSF}$ to correlate with pain intensity, ${ }^{54}$ and levels of IL-6, IL-10, and IFN- $\gamma$ to correlate with radiological progression. ${ }^{55}$ However, cytokines are known to work in a complex hierarchical network, and most of them show pleiotropic, redundant, and synergetic actions, making full understanding of the balance very challenging. Therefore, a single biomarker may be insufficient, and it has been further proposed to use ratios of two cytokines or develop a multivariate "cytokine signature" that evaluates the pattern of several cytokines at one point in time. ${ }^{18,19}$

While the majority of pro-:anti-inflammatory cytokine ratios were higher in subjects presenting symptoms, multivariate analysis showed significant difference only for PMS. This may reflect the complexity of interactions among cytokines and encourage further studies to select and include more parameters, study different populations and age-groups, and examine varied health and disease conditions.

\section{Conclusion}

In conclusion, we did not observe significant direct correlations between vitamin $\mathrm{D}$ and the symptoms studied. Further, while some individual serum pro-inflammatory cytokine levels associated significantly with different symptoms under study, multivariate cytokine profiles showed an interesting possible association only with symptoms related to PMS. Stratifying the subjects based on their vitamin D levels showed similar association of individual cytokines and pro:anti-inflammatory cytokine ratios, but not with the multivariate pattern. While these data delineate the complexity of the cytokine network, they support a role for pro-inflammatory cytokines in mediating adverse symptoms.

We realize that this study has several limitations. We focused on cytokine levels in sera of adult women who appeared to be healthy, and it would be of great interest to do similar studies on patients with other chronic diseases and inflammatory conditions. It should also be highlighted that data presented do not imply or prove causation. As an increasing volume of research supports the potential role of vitamin D and cytokines in all physiological and pathological processes, further studies are needed to shed more light on their possible association in relation to symptoms in health and disease.

\section{Acknowledgment}

This research was fully funded by the Public Authority for Applied Education and Training (PAAET) project number BE-14-09.

\section{Disclosure}

The authors report no conflicts of interest in this work.

\section{References}

1. Wöbke TK, Sorg BL, Steinhilber D. Vitamin D in inflammatory diseases. Front Physiol. 2014;5:244.

2. Pludowski P, Holick MF, Pilz S, et al. Vitamin D effects on musculoskeletal health, immunity, autoimmunity, cardiovascular disease, cancer, fertility, pregnancy, dementia and mortality: a review of recent evidence. Autoimmun Rev. 2013;12(10):976-989.

3. Heaney RP. The vitamin D requirement in health and disease. J Steroid Biochem Mol Biol. 2005;97(1-2):13-19.

4. Wilkins CH, Sheline YI, Roe CM, Birge SJ, Morris JC. Vitamin D deficiency is associated with low mood and worse cognitive performance in older adults. Am J Geriatr Psychiatry. 2006;14(12):1032-1040.

5. Kjaergaard M, Waterloo K, Wang CE, et al. Effect of vitamin D supplement on depression scores in people with low levels of serum 25-hydroxyvitamin D: nested case-control study and randomised clinical trial. Br J Psychiatry. 2012;201(5):360-368.

6. Lucas RM, Ponsonby AL. Considering the potential benefits as well as adverse effects of sun exposure: can all the potential benefits be provided by oral vitamin D supplementation? Prog Biophys Mol Biol. 2006;92(1):140-149. 
7. Holick MF. Vitamin D: importance in the prevention of cancers, type 1 diabetes, heart disease, and osteoporosis. Am J Clin Nutr. 2004;79(3): 362-371.

8. Holick MF. The vitamin D deficiency pandemic and consequences for nonskeletal health: mechanisms of action. Mol Aspects Med. 2008;29(6): 361-368.

9. Arabi A, El Rassi R, Fuleihan G. Hypovitaminosis D in developing countries: prevalence, risk factors and outcomes. Nat Rev Endocrinol. 2010;6(10):550-561.

10. Azizieh F, Alyahya KO, Raghupathy R. Association between levels of vitamin D and inflammatory markers in healthy women. J Inflamm Res. 2016;9:51-57.

11. White JH. Vitamin D signaling, infectious diseases, and regulation of innate immunity. Infect Immun. 2008;76(9):3837-3843.

12. Hewison M. Vitamin D and immune function: an overview. Proc Nutr Soc. 2012;71(1):50-61.

13. Dinarello CA. Proinflammatory cytokines. Chest. 2000;118(2):503-508.

14. Myers JS. Proinflammatory cytokines and sickness behavior: implications for depression and cancer-related symptoms. Oncol Nurs Forum. 2008;35(5):802-807.

15. Pine SR, Mechanic LE, Enewold L, et al. Increased levels of circulating interleukin 6, interleukin 8, C-reactive protein, and risk of lung cancer. J Natl Cancer Inst. 2011;103(14):1112-1122.

16. Kaminska J, Kowalska MM, Nowacki MP, Chwalinski MG, Rysinska A, Fuksiewicz M. CRP, TNF $\alpha$, IL-1ra, IL-6, IL-8 and IL-10 in blood serum of colorectal cancer patients. Pathol Oncol Res. 2000;6(1):38-41.

17. Zhang JM, An J. Cytokines, inflammation, and pain. Int Anesthesiol Clin. 2007;45(2):27-37.

18. Burska A, Boissinot M, Ponchel F. Cytokines as biomarkers in rheumatoid arthritis. Mediators Inflamm. 2014;2014:545493.

19. Davis JM 3rd, Knutson KL, Strausbauch MA, et al. Analysis of complex biomarkers for human immune-mediated disorders based on cytokine responsiveness of peripheral blood cells. J Immunol. 2010;184(12): 7297-7304.

20. de la Jara GT, Pécoud A, Favrat B. Musculoskeletal pain in female asylum seekers and hypovitaminosis D3. BMJ. 2004;329(7458):156-157.

21. Arvold DS, Odean MJ, Dornfeld MP, et al. Correlation of symptoms with vitamin $\mathrm{D}$ deficiency and symptom response to cholecalciferol treatment: a randomized controlled trial. Endocr Pract. 2009;15(3):203-212.

22. Huang W, Shah S, Long Q, Crankshaw AK, Tangpricha V. Improvement of pain, sleep, and quality of life in chronic pain patients with vitamin D supplementation. Clin J Pain. 2013;29(4):341-347.

23. Straube S, Derry S, Straube C, Moore RA. Vitamin D for the treatment of chronic painful conditions in adults. Cochrane Database Syst Rev. 2015;(5):CD007771.

24. Gominak SC, Stumpf WE. The world epidemic of sleep disorders is linked to vitamin D deficiency. Med Hypotheses. 2012;79(2):132-135.

25. Okun ML, Luther JF, Wisniewski SR, Wisner KL. Disturbed sleep and inflammatory cytokines in depressed and nondepressed pregnant women: an exploratory analysis of pregnancy outcomes. Psychosom Med. 2013;75(7):670-681.

26. Krueger JM, Majde JA, Rector DM. Cytokines in immune function and sleep regulation. Handb Clin Neurol. 2011;98:229-240.

27. Clinton JM, Davis CJ, Zielinski MR, Jewett KA, Krueger JM. Biochemical regulation of sleep and sleep biomarkers. J Clin Sleep Med. 2011; 7(5 Suppl):S38-S42.

28. Ryu A, Kim TH. Premenstrual syndrome: a mini review. Maturitas. 2015;82(4):436-440.

29. Bertone-Johnson ER, Chocano-Bedoya PO, Zagarins SE, Micka AE, Ronnenberg AG. Dietary vitamin D intake, 25-hydroxyvitamin D3 levels and premenstrual syndrome in a college-aged population. J Steroid Biochem Mol Biol. 2010;121(1-2):434-437.

30. Thys-Jacobs S, McMahon D, Bilezikian JP. Cyclical changes in calcium metabolism across the menstrual cycle in women with premenstrual dysphoric disorder. J Clin Endocrinol Metab. 2007;92(8): $2952-2959$.
31. Bertone-Johnson ER, Ronnenberg AG, Houghton SC, et al. Association of inflammation markers with menstrual symptom severity and premenstrual syndrome in young women. Hum Reprod. 2014;29(9):1987-1994.

32. Puder JJ, Blum CA, Mueller B, De Geyter C, Dye L, Keller U. Menstrual cycle symptoms are associated with changes in low-grade inflammation. Eur J Clin Invest. 2006;36(1):58-64.

33. Atherton K, Berry DJ, Parsons T, Macfarlane GJ, Power C, Hyppönen E. Vitamin D and chronic widespread pain in a white middle-aged British population: evidence from a cross-sectional population survey. Ann Rheum Dis. 2009;68(6):817-822.

34. McBeth J, Pye SR, O’Neill TW, et al. Musculoskeletal pain is associated with very low levels of vitamin D in men: results from the European Male Ageing Study. Ann Rheum Dis. 2010;69(8):1448-1452.

35. Warner AE, Arnspiger SA. Diffuse musculoskeletal pain is not associated with low vitamin D levels or improved by treatment with vitamin D. J Clin Rheumatol. 2008;14(1):12-16.

36. Armstrong DJ, Meenagh GK, Bickle I, Lee AS, Curran ES, Finch MB. Vitamin D deficiency is associated with anxiety and depression in fibromyalgia. Clin Rheumatol. 2007;26(4):551-554.

37. Uçeyler N, Häuser W, Sommer C. Systematic review with meta-analysis: cytokines in fibromyalgia syndrome. BMC Musculoskelet Disord. 2011;12:245.

38. Alyahya K, Lee WT, Al-Mazidi Z, Morgan J, Lanham-New S. Risk factors of low vitamin D status in adolescent females in Kuwait: implications for high peak bone mass attainment. Arch Osteoporos. 2014;9:178.

39. Lamberg-Allardt C, Brustad M, Meyer HE, Steingrimsdottir L. Vitamin D: a systematic literature review for the 5 th edition of the Nordic Nutrition Recommendations. Food Nutr Res. 2013;57:22671.

40. Pérez F, Granger BE. IPython: a system for interactive scientific computing. Comput Sci Eng. 2007;9(3):21-29.

41. Baringhaus L, Franz C. On a new multivariate two-sample test. J Multivar Anal. 2004;88(1):190-206.

42. RStudio [software]. 2016. Available from: https://www.rstudio.com. Accessed January 25, 2017.

43. Tawar S, Bhatia SS, Ilankumaran M. Mental health, are we at risk? Indian J Community Med. 2014;39(1):43-46.

44. Husain N, Chaudhry N, Rhouma A, Sumra A, Tomenson B, Waheed W. Validation of the self-reporting questionnaire (SRQ 20) in British Pakistani and white European population in the United Kingdom. J Affect Disord. 2016;189:392-396.

45. Peek MK, Ray L, Patel K, Stoebner-May D, Ottenbacher KJ. Reliability and validity of the SF-36 among older Mexican Americans. Gerontologist. 2004;44(3):418-425.

46. Zhang Y, Qu B, Lun S, Guo Y, Liu J. The 36-item short form health survey: reliability and validity in Chinese medical students. Int J Med Sci. 2012;9(7):521-526.

47. Wu S, Wang R, Zhao Y, et al. The relationship between self-rated health and objective health status: a population-based study. BMC Public Health. 2013;13:320.

48. Jepsen R, Dogisso TW, Dysvik E, Andersen JR, Natvig GK. A crosssectional study of self-reported general health, lifestyle factors, and disease: the Hordaland health study. PeerJ. 2014;2:e609.

49. Vézina-Im LA, Godin G, Couillard C, Perron J, Lemieux S, Robitaille J. Validity and reliability of a brief self-reported questionnaire assessing fruit and vegetable consumption among pregnant women. BMC Public Health. 2016;16:982.

50. Patrick DL, Cheadle A, Thompson DC, Diehr P, Koepsell T, Kinne S. The validity of self-reported smoking: a review and meta-analysis. Am J Public Health. 1994;84(7):1086-1093.

51. Spiro A, Buttriss JL. Vitamin D: An overview of vitamin D status and intake in Europe. Nutr Bull. 2014;39(4):322-350.

52. Fardellone P, Séjourne A, Paccou J, Goëb V. Bone remodelling markers in rheumatoid arthritis. Mediators Inflamm. 2014;2014:484280.

53. Miao J, Zhang K, Lv M, et al. Circulating Th17 and Th1 cells expressing CD161 are associated with disease activity in rheumatoid arthritis. Scand J Rheumatol. 2014;43(3):194-201. 
54. Davis JM, Matteson EL. [Cytokine biomarkers and the promise of personalized therapy in rheumatoid arthritis]. Reumatol Clin. 2009;5(4): 143-146. Spanish.
55. Tukaj S, Kotlarz A, Jóźwik A, et al. Cytokines of the Th1 and Th2 type in sera of rheumatoid arthritis patients; correlations with anti-Hsp40 immune response and diagnostic markers. Acta Biochim Pol. 2010;57(3):327-332.

\section{Publish your work in this journal}

The Journal of Inflammation Research is an international, peer-reviewed open access journal that welcomes laboratory and clinical findings on the molecular basis, cell biology and pharmacology of inflammation including original research, reviews, symposium reports, hypothesis formation and commentaries on: acute/chronic inflammation; mediators of inflammation; cellular processes; molecular mechanisms; pharmacology and novel anti-inflammatory drugs; clinical conditions involving inflammation. The manuscript management system is completely online and includes a very quick and fair peer-review system. Visit http://www.dove press.com/testimonials.php to read real quotes from published authors. 